\title{
Justicia Ambiental, Capitaloceno y Cambio Climático en Brasil y Colombia. Reflexión crítica
}

\section{Environmental Justice, Capitalocene and Climate Change in Brazil and Colombia.Critical reflection}

\author{
João Marcelo Quintiliano Ramos ${ }^{1}$; Iván Darío Loaiza Campiño² \\ 1 Licenciado en Ciencias Ambientales, Unirio, Rio de Janeiro, Rio de Janeiro, Brasil - \\ joramos750@gmail.com,/https://orcid.org/0000-0003-2944-4396 \\ 2 Ing. Agrónomo. Msc. Ciencias Biológicas. Docente Cátedra Ambiental Gonzalo Palomino y de la Ing. en Agroecología. \\ Grupo de investigación GEBIUT., Universidad del Tolima, Ibagué, Tolima, Colômbia- idloaizac@ut.edu.co \\ ,https://orcid.org/0000-0003-4873-724X
}

\section{Palavras-chave: \\ Colonialidad 1. \\ Comunidades 2. Racismo 3. Agronegocio 4. \\ Desarrollo 5.}

Keywords:

Coloniality 1.

Communities 2.

Racism 3. Agribusiness4. Developing 5.
RESUMO: La crisis climática y ecológica presenta un cuadro crítico en todo el mundo. No son suficientes los acuerdos climáticos establecidos a lo largo de estos años como forma de mitigación, adaptación y prevención a los impactos resultado de los efectos del cambio climático como iniciativas entre otras, del programa de Naciones Unidas para el ambiente. Científicos e investigadores lo consideran como una nueva Era Geológica, o Antropoceno. Justificando la responsabilidad humana, como un todo en relación a los cambios globales y se considera la interferencia humana como aspecto que prevalece en la crisis ambiental. Dentro de este espectro, la investigación vislumbra posicionar una reflexión crítica en relación a los actores realmente responsables, ubicando el Capitaloceno como una forma de perpetuar un sistema económico que reproduce desigualdades y privilegios. Diferentes organizaciones impulsan la llamada economía verde, como estrategia para ocultar el verdadero papel de las corporaciones en esta crisis y hacer compatible el capitalismo con la ecología. Estos factores se relacionan además con los conceptos de justicia ambiental y racismo ambiental, reflejados en las condiciones que enfrenta esta crisis, siendo un factor determinante de opresion en los pueblos principalmente de personas negras y pobres. En las tierras del sur global, el capitalismo se potencia también con otros sistemas de opresión y patriarcado que perpetúan esta larga historia de violencia y despojo.

ABSTRACT: Climate and ecological crisis presents a critical picture throughout the world. The climate agreements established throughout these years as a form of mitigation, adaptation and prevention of the impacts resulting from the effects of climate change as initiatives among others, of the United Nations program for the environment are not enough. Scientists and researchers consider it as a new Geological Age, or Anthropocene. Justifying human responsibility as a whole in relation to global changes and human interference is considered as prevailing in the environmental crisis. Within this spectrum, the research envisages positioning a critical reflection in relation to the really responsible actors, placing the Capitalocene as a way to perpetuate an economic system that reproduces inequalities and privileges. Different organizations promote the so-called green economy, as a strategy to hide the true role of corporations in this crisis and make capitalism compatible with ecology. These factors are also related to the concepts of environmental justice and environmental racism, reflected in the conditions facing this crisis, being a determining factor of oppression in the villages mainly of black and poor people. In the lands of the global south, capitalism is also enhanced by other systems of oppression and patriarchy that perpetuate this long history of violence and dispossession. 


\section{Justicia ambiental}

Definir la justicia ambiental no es tarea fácil, ya que existen muchas posibles definiciones. La justicia ambiental es una conceptualización compleja, ya que combina factores sociales, políticos y económicos, y son todos estos factores los que contribuyen a la relación positiva, ya evidenciada, entre las comunidades más desfavorecidas y los efectos ambientales negativos (Helfand y Peyton 1999, citado por Arriaga, 2009). Se entiende de manera amplia la Justicia Ambiental como la preocupación por la capacidad de soporte del ambiente ante la presión de las actividades humanas (Pérez, 2018).

El término de justicia ambiental es, indudablemente, de carácter distributivo ya que su argumento básico se refiere a la preocupación que se deriva sobre cómo los impactos ambientales, positivos y negativos, se distribuyen entre los distintos miembros de la sociedad. Las cuestiones de procedimiento también son centrales a la justicia ambiental: aquellas relacionadas con la justicia y la equidad en el acceso a los procesos de toma de decisiones ambientales; así como en el acceso a los derechos y recursos del derecho ambiental (Arriaga y Pardo 2009).

Arriaga y Pardo (2009) citan a Bryant (1995), para afirmar que la justicia ambiental "se refiere a las normas y los valores culturales, reglas, reglamentos, conductas, políticas y decisiones de apoyo a comunidades sostenibles, donde la gente puede interactuar con la confianza de que su entorno es seguro, cuidado y protegido". En lo que la mayoría de las definiciones del concepto de justicia ambiental coinciden es en que el término no sólo es reactivo a los "males" ambientales, sino lo que es más importante, también es proactivo de la consecución de las "bondades" o beneficios ambientales, como son las comunidades sostenibles y el disfrute de una mayor calidad de vida (Agyeman y Evans 2004).

Según Martínez Alier la justicia ambiental se trata de una corriente que viene desde los países "pobres" -aunque también reconoce raíces en los grupos menos favorecidos de los países ricos-: la Justicia Ambiental, el Ecologismo Popular o el Ecologismo de los Pobres. Para Martínez Alier, la ética de esta corriente nace de una demanda de justicia social contemporánea entre humanos. Además de que el crecimiento económico implica impactos al ambiente, esta corriente enfatiza el desplazamiento geográfico tanto de las fuentes de recursos como de los sumideros de residuos. En este sentido, ciertas "fronteras": la "frontera del cobre" y la "frontera del oro", por ejemplo, avanzan hacia nuevos territorios. "Esto crea 
impactos que no son resueltos por políticas económicas o cambios en la tecnología, y por tanto caen desproporcionadamente sobre algunos grupos sociales que muchas veces protestan y resisten (aunque tales grupos no suelen llamarse ecologistas)" (Gutiérrez, 2014, citando a Martínez Alier, 2004, p. 27).

El movimiento por la justicia ambiental surge en Estados Unidos en la década de 1980, como resultado de un movimiento popular en contra de la instalación de un vertedero de 120 millones de libras de tierra con policlorobifenilos en la provincia de Warren, Carolina del Norte, la cual era habitada en su mayoría por gente de color, quienes al verse rebasados por la decisión gubernamental, optaron por las protestas de resistencia pacífica para que se respetase su derecho a la salud y a un ambiente sano, comenzando a acuñarse el término de justicia ambiental. A partir de este caso surge otra corriente de pensamiento donde se pone de manifiesto además del impacto sobre la naturaleza, las intenciones de verter estas sustancias contaminantes en zonas donde habitan principalmente comunidades negras, una clara muestra de diferencias de poder, entre las multinacionales que produjeron los contaminantes, las poblaciones de mayor poder adquisitivo, quienes necesitan deshacerse de la contaminación y otros que son considerados inferiores (clara visión colonialista) apareciendo así el Racismo Ambiental como nueva categoría de análisis (Ramírez et al, 2015).

\section{Efectos de la colonialidad y la crisis climática.}

Durante mucho tiempo, la colonialidad tomó la forma de una nube que se cernía sobre las tierras brasileñas y latinoamericana, manteniendo los efectos del colonialismo hasta nuestros días. Hoy, el proceso de colonialidad mantiene a los pueblos tradicionales y sus descendientes a la deriva, en el contexto de una sociedad altamente excluyente. El estado de Río de Janeiro puede ser una región con un alto PIB; Gigante por naturaleza en su refugio: banco de arena, mar, manglar y selva tropical atlántica. Sin embargo, persiste el estigma del colonialismo y los egeítas de su colonialidad visibles en la profunda desigualdad social que abraza al estado.

La desigualdad social es notable en la distribución de la población que habita las áreas más remotas del estado a las ocupaciones irregulares, agrupadas en los centros urbanos se identifican como el tema "suburbano", "barrio marginal" y "periférico", todos estos nombres tienen características auténticas y, sin embargo, muy comunes, como el color, la raza y la clase social, que denotan los efectos de la colonialidad en las personas. 
Ensino, Saúde e Ambiente - Número Especial, pp. 389-411, Junho. 2020

La perspectiva de la colonialidad en la dimensión territorial del Estado de Río de Janeiro, tangente a los aspectos reconocidos del proceso de cambio climático, muestra que hay un porcentaje mínimo de la población, reconocida como blanca, alfabetizada, con poder económico, capaz de sostener la alta especulación inmobiliaria. El "hombre blanco" se caracteriza por la figura eurocéntrica que posee poder económico y razón intelectual, razón capaz de invalidar cualquier tipo de cosmologías, fe y conocimiento tradicional, pudiendo transformar el sujeto no blanco (negro e indígena) en un actor criminal por los motivos ya mencionados. La raza negra está sujeta a la inseguridad de la vivienda vinculada a la vivienda en los barrios marginales junto con la violencia policial. Sirve como una herramienta para un proceso de estado genocida, a través del cual el concepto político crítico de raza es sostenido por estos pueblos no blancos. "En otras palabras, la raza no es más que un concepto establecido como un instrumento de clasificación social y política" (CATARINO, 2016, p. 25).

Las bases del racismo, desde la perspectiva de la modernidad, la colonialidad y el capitalismo convergen con los orígenes de la degradación ambiental y las desigualdades sociales que afectan a las poblaciones indígenas, campesinas y africanas en Brasil y Colombia. Los agronegocios, la construcción de presas y la especulación inmobiliaria, entre otros factores, han contribuido a perpetuar el racismo ambiental y la concentración de estas poblaciones en áreas de sacrificio (BARROZO, L.A.; SANCHÉZ, C., 2015).

\section{El concepto de racismo ambiental}

El racismo ambiental consiste en sacrificar una población étnica predominantemente no blanca, como los pueblos negros e indígenas, que viven oprimidos por el estado, para socavar su entorno de vida, junto con su bajo nivel socioeconómico. Habita en un lugar particular, considerablemente distante de los centros urbanos, donde este territorio está marcado por la opresión ambiental (KASSIADOU, A. et al. 2018) para que cualquier nivel de alto riesgo pueda dañar gravemente El bienestar, la salud y la dignidad de esa población.

El factor raza se correlacionó más fuertemente con la distribución de ubicación de los desechos peligrosos que el factor de bajos ingresos. Fue a partir de esta investigación que el reverendo Benjamin Chavis utilizó por primera vez el término "racismo ambiental" para designar "la imposición desproporcionada - intencional o no intencional - de residuos peligrosos para las comunidades de color". En 1991, el Movimiento Negro celebró la Primera 
Cumbre Nacional de Liderazgo Ambiental de la Gente de Color (Bullard, 1996, CATARINO, 2016).

Según el mapa de injusticia ambiental, en el estado de Río de Janeiro, las regiones donde los municipios están más alejados de la gran metrópoli, es precisamente el entorno en el que está habitada la mayoría de la población negra (Disponible en: https://racismoambiental.net.br/2018/07/26/mapa-de-facing- to-racismo /,). Siendo estas regiones se considera lo que se ha llamado en la literatura como "zonas de sacrificio" (ACSELRAD, H. 2000), varias empresas altamente contaminantes, con permiso del gobierno, se encuentran en estos lugares. No solo la contaminación en sí misma, sino las diversas formas de descuido de las políticas públicas, como la salud y la vivienda, contribuyen a la configuración del racismo ambiental y el no derecho a la ciudad. Dado que la mayoría de la población pobre y negra no puede permitirse el lujo de vivir en una vivienda digna, están condicionados a habitar ocupaciones irregulares, mejor conocidas como barrios marginales.

Como una de las expresiones del movimiento ambiental, el movimiento de justicia ambiental nació en los Estados Unidos alrededor de la década de 1960, con fuertes lazos con la lucha por los derechos civiles de Martin Luther King, y se organizó para combatir casos de "racismo ambiental", por ejemplo, en áreas de contaminación del aire, centros municipales de eliminación de desechos, desechos tóxicos y otros peligros ambientales concentrados cerca de los barrios más pobres o habitados por minorías raciales. La lucha por la justicia ambiental también incluye movimientos campesinos (Martínez-Alier, 2007).

\section{Antropoceno y Capitaloceno, los generadores de injusticia ambiental}

\section{1 ¿Qué es el Atropoceno?}

Los planteamientos en torno al Antropoceno son claves para entender la injusticia ambiental, pues destacan el papel de los humanos en las transformaciones históricas de lo biofísico y presentan la necesidad de incluir a la naturaleza en los análisis históricos y en otras ciencias sociales y humanas como parte de la interrelación con los humanos. Este cambio de perspectiva ocurre a partir de un análisis crítico del eje conceptual de la dualidad naturaleza y cultura, para replantear estas categorías como recíprocas. Esta consideración ha tenido lugar en la antropología y en las ciencias sociales y humanas desde la década de 1970. Sin embargo, en la actualidad ha alcanzado también a las ciencias naturales y a maneras disciplinarias de producir conocimiento, al igual que a los debates públicos y las políticas públicas 
relacionadas con lo global-local. Su trascendencia se debe en parte a que el concepto emerge en las ciencias geológicas (ciencias de la tierra) y tiene aceptación en diversos ámbitos académicos, aun entre los teóricos críticos de las ciencias humanas (Ulloa, 2017).

Si bien el concepto de Antropoceno es útil para marcar un cambio en la historia geológica causado por las actividades humanas y mostrar los impactos negativos sobre el clima, carece de la precisión suficiente como para no ser manipulado y justificar más de lo mismo. Quienes ven en el Antropoceno la culminación del potencial humano para dominar y controlar la naturaleza difunden la idea de que el cambio climático y el calentamiento global no son sino males menores que pueden ser resueltos con más tecnología (visión tecnocentrista). Para ellos la humanidad, lo mismo que los ecosistemas, tiene la capacidad de adaptarse a cualquier nuevo escenario, por lo que no es necesario reducir el consumo material y el crecimiento industrial, todo lo contrario, "el planeta es más productivo que antes de ser alterado por los humanos", y si fuimos capaces de adaptarnos a problemas anteriores, lo volveremos a lograr (Cano, 2017).

En torno a esta noción está la idea de que el Antropoceno ha sido causado por nuestra naturaleza humana, que es egoísta, individualista, nos impide ver más allá del corto plazo y nos impulsa a la conquista de la naturaleza. De esta manera, si todos tenemos la misma naturaleza, todos somos responsables de los problemas del Antropoceno. Una postura en consonancia con los Gobiernos y las empresas que prometen en cada crisis generar más riqueza y más consumo; agentes que mediante el uso de mecanismos legales y de propaganda hacen a todos responsables de la crisis actual (Vansintjan, 2016, citado por Cano, 2017).

\section{2 ¿Qué es El Capitaloceno?}

El concepto de Capitaloceno, según el mexicano Omar Cano Ramirez, alude a que, si bien: "fue con la Revolución industrial que comenzó la quema de combustibles fósiles y la expulsion de gases de efecto invernadero a la atmósfera, esa revolución no se dió en un vacio social. Al contrario se desarrolló en un sistema económico que requiere e impulsa la innovación tecnocientífica para movilizar mercancias lo más rápido posible a distancias crecientes" (Madrid, 2019).

Podemos aseverar que en el Capitaloceno, la crisis medioambiental que afecta a toda las especies vivas del planeta no fue impulsada e incentivada anónimamente y por todos, por algo que se puede explicar como un desequilibrio del poder social. Siguiendo a este autor Cano Ramírez aclara: "no todos han tenido el mismo poder social para influir sobre la 
Ensino, Saúde e Ambiente - Número Especial, pp. 389-411, Junho. 2020

estructura económica: desde los esclavos usados para el desarrollo del capitalismo europeo, pasando por las comunidades indígenas casi exterminadas y marginadas, hasta la gente que desde el siglo XX sufre la miseria y explotación laboral", la mayor parte de la población no ha contribuido en absoluto a la crisis de carácter antropógena que occidente nos achaca (Madrid, 2017).

En las tierras del sur global, el capitalismo se potencia con otros sistemas de opresión como el colonialismo y el patriarcado, que perpetúan una larga historia de violencia. Actualmente este sistema se intensifica con un nuevo ciclo extractivista, donde el saqueo de estas tierras nutre las dinámicas globales de acumulación. Son tiempos difíciles, megaemprendimientos mineros, agroindustriales, forestales y de pesca de arrastre redefinen los territorios y gestionan con complejas estrategias de violencia, material y simbólica, las relaciones sociales y subjetividades (Colectivo El Kintral, 2018).

Para Toledo (2019), El cambio climático no debe entonces atribuirse al mero hecho de que el planeta esté poblado por 7 mil millones, sino al reducido número de personas (uno por ciento) que controlan los medios de producción y deciden cómo se ha de usar la energía. Se trata entonces de actuar contra el capital fósil. En contraposición con lo anterior, el mismo autor afirma que todo el aparato del sistema opera para que los ciudadanos no reconozcan y adopten esa posición. En lenguaje diplomático: se trata de no politizar la situación. No sólo los negacionistas de la crisis ecológica y climática actúan en esa línea, sino también entidades enteras como el Programa de Naciones Unidas para el Medio Ambiente (Pnuma), que desde 2012 impulsa con mucha fuerza la llamada economía verde, una estrategia para ocultar el papel de las corporaciones y hacer compatible el capitalismo con la ecología, o la FAO, que a regañadientes ha aceptado hasta recientemente a la agroecología y al campesinado como opción ante los sistemas destructivos agroindustriales, que es la vía capitalista en la agricultura. En el ocultamiento antropogénico participan también científicos conservadores. En México, como hemos señalado, existe el caso de que conocidas figuras de la ecología encabecen las campañas de lavado verde (green-washing) de las mayores corporaciones como Coca Cola, Volkswagen, Cemex, Bimbo, Telmex, Grupo México (https://bit.ly/2YYZtC7) e impulsen conceptos como el de capital natural, que apuesta por el carácter virtuoso de la mercantilización de la naturaleza. En suma, hoy resulta cada vez más difícil negar que vivimos inmersos en una nueva era geológica, que más que antropoceno debe llamarse capitaloceno, y que debemos salir de ella lo más rápido posible, antes de que el destino nos rebase (Toledo, 2019). 
Ensino, Saúde e Ambiente - Número Especial, pp. 389-411, Junho. 2020

\section{Injusticia y Racismo Ambiental del discurso del cambio climático}

El Cambio Climático en una verdad a medias, existe un gran número de científicos, periodistas, políticos economistas, entre otros, que afirman que el cambio climático se debe a las altas concentraciones en la atmósfera de gases efecto invernadero, entre ellos y el más importante según la mayoría de científicos el $\mathrm{CO}_{2}$ derivado de la combustión de fuentes de energía fósil. Sin embargo es triste ver como se atribuye a la especie humana en general, de manera indiferenciada las emisiones de $\mathrm{CO}_{2}$.

Toleto (2019), afirma por ejemplo que, el pecado mayor del ambientalismo, el conjunto de movimientos en defensa de la naturaleza y sus autores, fue habernos hecho creer que los culpables de la destrucción del mundo natural éramos todos los seres humanos sin excepción. Esta idea, alimentada por la visión estrecha e incompleta de la biología, predominó durante décadas, y si bien sirvió para un saludable cambio de conducta a escalas individual, familiar y grupal, también operó como eficaz mecanismo que desvió la atención de los verdaderos culpables. En la arena científica, la cúspide de esta concepción se alcanzó con la adopción en la jerga académica del concepto de antropoceno, formulada por Paul Crutzen, premio Nobel de Química y uno de los estudiosos más destacados de la atmósfera. El antropoceno quedó definido como una nueva era geológica en la que la acción humana (la civilización moderna e industrial) se ha convertido en una nueva fuerza capaz de alterar los mayores procesos y ciclos del planeta. Hubo que esperar el desarrollo y proliferación de una ecología política para cuestionar mediante evidencias bien documentadas, las limitaciones de esa visión. A ello contribuyeron numerosos autores que fueron develando los mecanismos de la devastación de manera crítica. Por ejemplo, en 2015, la mitad de las emisiones totales de $\mathrm{CO}_{2}$ fueron responsabilidad de 10 por ciento de la población con más riqueza -700 millones de personas-, mientras la mitad de la población mundial -3 mil 500 millones- sólo generó 10 por ciento de las emisiones. Aún peor: según Oxfam, las emisiones de carbono de uno por ciento más rico son 30 veces mayores que las de 50 por ciento más pobre. Los agentes más contaminantes en la historia son las corporaciones petroleras, gaseras y cementeras. Como vimos en un artículo anterior (https://bit.ly/2uVIEu6), entre 1751 y 2010, tan sólo 90 corporaciones emitieron 63 por ciento del total de gases de efecto invernadero (Toledo, 2019).

Borrás (2017) hace otro aporte para entender la injusticia ambiental a través de la injusticia climática, afirmando que no solo la generación del problema climático, sino también el modelo de desarrollo, han sido el origen de la injusticia: es decir, el desarrollo desigual entre los países provoca que cerca de tres cuartas partes del exceso de $\mathrm{CO}_{2}$ acumulado en la 
Ensino, Saúde e Ambiente - Número Especial, pp. 389-411, Junho. 2020

atmósfera hasta ahora provenga de los países más industrializados. Por ejemplo, Kiribati, un pequeño estado insular situado en el océano Pacífico, producía en 2011 0,6 toneladas de $\mathrm{CO}_{2}$ per cápita, mientras que España generaba 5,8 y Estados Unidos 16,8.

\section{Casos de racismo e injusticia climática en Colombia y Brasil.}

\subsection{Cambio climático y su efecto en la aparición de enfermedades en Brasil y Colombia}

En 2009, en Brasil, se estableció la Política Nacional de Cambio Climático, que ratifica el compromiso del país con la Convención Marco de las Naciones Unidas sobre el Cambio Climático. "La política mencionada se basa en la Ley $N^{\circ} 12.187,2009$. Señala los ejes de: (I) mitigación; (II) vulnerabilidad, impacto y adaptación, (III) investigación y desarrollo; y (IV) capacitación y difusión. ”(BRASIL, 2009, p.26).

El Plan Nacional de Cambio Climático fue creado a partir de varias coaliciones, incluida la Comisión Interministerial sobre Cambio Climático, el Foro Brasileño sobre Cambio Climático, la III Conferencia Nacional de Medio Ambiente y los Foros Estatales sobre Cambio Climático. Dentro de la lógica de un país en desarrollo, Brasil alinea el plan de adaptación con la estrategia de desarrollo. En este sesgo, los objetivos principales se elaboran en el documento, como el aumento de la tecnología para la eficiencia energética. Otro problema es la contención de la deforestación desenfrenada en el territorio amazónico, con el apoyo del Fondo Amazonas, configurado como un fondo para recaudar recursos de los países del Anexo I, con el propósito de preservar y restaurar el medio ambiente amazónico. Recordando que el Amazonas tiene un papel importante en el mundo como sumidero de $\mathrm{CO} 2$, combatiendo el calentamiento global.

El objetivo general del Plan Nacional de Cambio Climático es identificar, planificar y coordinar las acciones y medidas que se pueden tomar para mitigar las emisiones de gases de efecto invernadero generadas en Brasil, así como las necesarias para adaptar la sociedad a los impactos que ocurren debido al cambio climático (BRASIL, 2009, p.26).

Citando los objetivos generales del Plan Nacional de Cambio Climático, el documento aborda brevemente: formas de ser optimizado en la economía; invertir en matriz de energía renovable; aumentar la producción y establecer un mercado exclusivo para biocombustibles; reducción de la deforestación ilegal; eliminar la pérdida de área ya deforestada; visualizar los impactos causados por el cambio climático; además de formular soluciones a través de la investigación científica y el desarrollo tecnológico. 
Ensino, Saúde e Ambiente - Número Especial, pp. 389-411, Junho. 2020

En relación con el último factor mencionado, destacando como la base de este estudio, el documento presenta: "[...] fortalecer las acciones intersectoriales destinadas a reducir las vulnerabilidades de las poblaciones."(BRASIL, 2009, p.27). Refiriéndose al fragmento mencionado, el plan contempla contemplar acciones entre sectores para reducir los factores que causan vulnerabilidades en las poblaciones. Se entiende que la causa de la vulnerabilidad de una población está vinculada a los tres pilares: poder económico, salud y educación, que comentaremos más adelante.

Desde 1950, las regiones del sudeste y sur de Brasil han experimentado un aumento en la frecuencia de los días lluviosos intensos, así como un aumento en la frecuencia de los sofocos y días secos consecutivos, lo que significaría fuertes lluvias concentradas en unos pocos días con períodos secos entre eventos lluviosos; En el noreste, la frecuencia de días secos consecutivos aumenta en longitud e intensidad (MAGRIN et. al., 2014).

El riesgo asociado con estos desastres depende en gran medida de las condiciones socioeconómicas y culturales de las poblaciones expuestas, así como de la forma en que las instituciones estatales manejan los desastres; en otras palabras, el riesgo depende de la vulnerabilidad.

Con el fin de obtener un análisis preciso de los datos de vulnerabilidad climática en el estado de Río de Janeiro, se sintetizarán los datos recopilados y procesados por Fiocruz, publicados en el capítulo 9 del libro "Modelado climático y vulnerabilidades sectoriales al cambio climático en Brasil", titulado como 'Índice de vulnerabilidad al cambio climático en municipios del estado de Río de Janeiro' (MARINHO, D.P. et. al., 2014) y el sitio web propio de la institución, en términos más generales, como "Mapa de la vulnerabilidad de la población de los municipios del estado de Río de Janeiro ante el cambio climático", en 2014. (Disponible en: <http://www.fiocruz.br/ioc/cgi/cgilua.exe/sys/start.htm?sid=122,>)

El documento 'Índice de vulnerabilidad al cambio climático en los municipios del estado de Río de Janeiro' comienza conceptualizando la vulnerabilidad, basándose en la descripción de los informes de evaluación del IPCC (AR) de 2007 a 2013. AR4 (2007) conceptualiza como resultado exposición, sensibilidad y capacidad de adaptación. En AR5 (2013), se aclaran los mismos conceptos, aunque la capacidad de adaptación se trata como un concepto de riesgo. Entre ellos, el "factor de peligro" con respecto al cambio climático futuro se ha agregado y se estudiará como el Índice de Escenario Climático (ICC); La exposición se refiere a la vulnerabilidad en el tema epidemiológico y su capacidad de adaptación está vinculada a los análisis sociales. 
Ensino, Saúde e Ambiente - Número Especial, pp. 389-411, Junho. 2020

El Índice de Vulnerabilidad Municipal (MIV) tiene una característica multidimensional y puede combinar factores ambientales, socioeconómicos y de salud. Su implementación fue una propuesta pionera en Brasil. La estructura del índice que se detallará más adelante se basa en un modelo esqueleto en el que el resultado final, IVM, consta de los siguientes subíndices: el ICC (Índice de escenario climático); el IVG (Índice de vulnerabilidad general); De este flujo provienen los del IVS (Índice de vulnerabilidad de la salud); IVSo (Índice de Vulnerabilidad Social) e IVAM (Índice de Vulnerabilidad Ambiental).

Para analizar el IVS (Índice de vulnerabilidad de la salud), según Marinho (et. al., 2014), se estudiaron cuatro tipos diferentes de enfermedades, cuyas variaciones de propagación están sujetas al escenario de cambio climático. Entre ellos se eligieron: dengue, leptospirosis, leishmaniasis cutánea estadounidense (ATL) y diarrea (que puede conducir a la muerte y se transmite fácilmente a través de factores ambientales). Los parámetros construidos a partir de la evaluación de la morbilidad y la mortalidad se hacen una atribución de pesos y sus cálculos.

La relación que cada enfermedad tiene con las anomalías climáticas proviene de varios eventos extremos que pueden ejemplificarse. Para el dengue, ya se está considerando una temporada de eventos de calor extremo, así como la contribución que brinda la estructura de la ciudad, como las islas de calor. Abren un espacio propicio para la propagación de la enfermedad, así como la reproducción a gran escala del vector Aedes aegypti. Siendo una situación desfavorable para el medio ambiente a través del cual viven las poblaciones marginadas con bajo desarrollo socioeconómico.

Según Aström (et al., 2011 apud MARINHO, D. P. et. Al. 2014), el efecto del clima en la aparición del dengue aún no está claro. Se sugiere que la distribución geográfica de los vectores del dengue esté limitada por las condiciones climáticas, aunque existen otros factores, como los factores socioeconómicos, que favorecen fuertemente la aparición de la enfermedad.

Los eventos hidrometeorológicos, como las precipitaciones extremas, se consideran responsables del movimiento de las masas de tierra y especialmente de las inundaciones, esto forma una base de propensión a la propagación de la leptospirosis por la dispersión de las ratas.

Levett (2001) afirma que los cambios en el clima y el comportamiento humano son capaces de alterar la epidemiología de la leptospirosis, y que los casos que siguen a varios ISSN 1983-7011 
episodios de inundaciones e inundaciones relacionados con El Niño han devuelto la atención del mundo a esta enfermedad. La explicación actual del efecto de las inundaciones y las inundaciones en la aparición de casos de leptospirosis está relacionada con la facilitación de la dispersión de ratas, principales huéspedes no humanos y su orina contaminada por bacterias patógenas (ÁVILA-PIRES, 2006).

El Índice de Vulnerabilidad Social (IVSo), tiene como objetivo un estudio del sujeto que ocupa las áreas delimitadas por los municipios. La investigación que trata sobre poblaciones vulnerables al cambio climático tiende a analizar en qué aspectos se enmarcan las poblaciones afectadas. Es cierto que el estado de Río de Janeiro concentra un nivel relativamente alto de PIB en la región metropolitana, en detrimento de las capas externas. Al ser un polo de desarrollo económico, con respecto a la inversión turística que tiene la ciudad de Río de Janeiro, la población que reside en él, tiene una condición económica favorable a su costo. Teniendo también en cuenta los grandes estratos populares que se asignan en los barrios bajos, buscando acceso a ingresos, salud y educación. Sin embargo, la vulnerabilidad social, de acuerdo con Barata (et al., 2011 apud MARINHO, DP et. Al. 2014), considera que los grupos sociales más vulnerables son los menos resistentes a los posibles impactos del cambio climático, como lluvias excesivas, inundaciones, resacas y problemas de salud.

Según Barata, el IVSo consta de seis dimensiones, 22 componentes y 50 indicadores. Describiendo brevemente las variables analizadas por municipio, las variables y sus subparticiones, dimensiones y componentes, respectivamente, se describen como: estructura familiar - cuidado y cuidado de niños, adolescentes y jóvenes, presencia del cónyuge, ausencia de desventaja física y ausencia de desventaja social; acceso al conocimiento: analfabetismo y educación; acceso al trabajo: disponibilidad de trabajo, calidad del trabajo y remuneración; disponibilidad de recursos - pobreza; desarrollo infantil: protección contra el trabajo temprano, acceso a la escuela, progreso escolar y mortalidad infantil y condiciones de vivienda: densidad de la propiedad, vivienda, acceso al suministro de agua, acceso al servicio de alcantarillado, acceso a recolección de basura, acceso a servicios de electricidad y acceso a bienes duraderos. "Los municipios con los valores IVSop más altos o más vulnerables fueron São Francisco de Itabapona (1.00), Varre-Sai (0.91), Trajano de Morais y Sumidouro (0.89), mientras que el vulnerables fueron Niterói (0.00) y Río de Janeiro (0.18). ”(MARINHO, DP et al., 2014).

Lo que respecta a Colombia llama la atención las escazas publicaciones realizadas sobre el efecto del cambio o la variabilidad climática sobre las enfermedades en humanos, en 
Ensino, Saúde e Ambiente - Número Especial, pp. 389-411, Junho. 2020

contraste por lo expuesto anteriormente en casos Brasileros. Los pocos trabajos publicados enfatizan en los efectos que ha tenido el fenómeno del Niño sobre las temperaturas y la precipitación y como estos influencian la densidad vectorial del Aedes aegypti. Según lo reportado estos efectos son reportados en otras partes del mundo, coincidiendo en los efectos de los cambios de temperatura sobre el desarrollo larvario.

Otras publicaciones encontradas hacen referencia a reportes de casos de hanta virus. Cárdenas y Malagón reportan que el fenómeno de Niño en 2008 y 2010 redundó en un aumento de Leismaniasis cutánea.

Malagón y colaboradores (2017) aportan datos adicionales para el análisis colombiano, en el caso de chikungunya, durante el 2015 se reportaron más de 200000 casos de los cuales el $14 \%$ presentó un cuadro de artritis crónica que afecta la funcionaliad de alrededor de 25000 personas en edad laboral (Pineda et al, 2016). Igualemente, se ha señalado al cambio climático como un factor determinante en la epidemis de zika en 2015 en latinoamerica y además de ser el principal virus en los Estados Unidos (Moreno y Turell, 2017).

En el caso de la enfermedad de chagas, se ha señalado al cambio climático como responsable del cambio en los ecotopos y el comportamiento de los vectores y reservorios de Tripanosoma cruci desplazándose a nuevas zonas lo cual ha aumentado la generación de brotes de enfermedad de chagas transmitidos por vía oral (Rueda et al 2014).

\subsection{EI Cambio climático y su efecto en las costas de Brasil y Colombia}

Volviendo al contexto del calentamiento global, este efecto proporciona una fusión considerable de los glaciares y los casquetes polares en general, lo que lleva a un aumento del nivel medio del mar. En dos sentidos, tenemos el calentamiento extremo en un sentido que proporciona un clima insoportable para la población de la ERJ, y en el otro, el aumento de NMM, que corresponde a otros eventos climáticos.

De la información proporcionada por el informe "Impactos, vulnerabilidades y adaptaciones en las regiones costeras", 2016, no hay datos suficientes para medir los cambios de NMM en Brasil. Sin embargo, durante décadas, algunos niveles han sido evaluados por investigadores, como el Instituto Nacional de Investigación Hidrográfica (INPH), Barbier (2015), Nicholls (et al., 2011 apud MARENGO, JA; SCARANO, FR, 2016), Tendencias en el NMM frente a la costa de Brasil fueron revisados por Neves y Muehe (1995), Mesquita 
Ensino, Saúde e Ambiente - Número Especial, pp. 389-411, Junho. 2020

(2003) y Muehe (2006), Losada (et. Al., 2013), Klein y Short (2016) y Silva (1992 apud MARENGO, JA; SCARANO F. R., 2016). Todos estos autores concluyeron que varios eventos extremos como las resacas e inundaciones tienen que ver con el aumento del nivel promedio del mar. "Las inundaciones, junto con las inundaciones, comprometen el drenaje de las zonas costeras, aumenta la frecuencia de las inundaciones y las inundaciones y disminuye la calidad del agua" (SANTOS, 2012 Apud: MARENGO, J. A .; SCARANO, F. R., 2016).

A través de esto, Silva (1992) identificó una variación de alrededor de 12,6 mm por año, de 1965 a 1986, en el territorio de Río de Janeiro. Belém (2007), concluyó en su investigación, un aumento del nivel del mar en tasas de 8 a 10 cm en la región sur del país. El mismo aumento es similar a otros puntos, incluido Río de Janeiro.

Según Belém (2007), varias ciudades de Brasil se encuentran en la situación en que el 60\% de la población vive a menos de 60 kilómetros de la costa (Río Grande-RS, Laguna-SC, Florianópolis-SC, Paranaguá-PR, Santos SP, Río de Janeiro-RJ, Vitoria-ES, Salvador-BA, Maceió-AL, Recife-PE, Sao Luis-MA, Fortaleza-CE, Belém-PA). El autor afirma que al comparar los diferentes puntos de la costa brasileña, la tendencia al aumento del nivel del mar es clara, pero a tasas variables de $8 \mathrm{~cm}$ en algunos puntos a $10 \mathrm{~cm}$ en el sur del país, teniendo en cuenta los datos de los últimos 10 años (MARENGO, J. A .; SCARANO, F. R., 2016).

Las costas colombianas según reporte de la revista Semana Sostenible (2017), enfrentan varios riesgos: En primer lugar el cambio en la temperatura superficial del mar, que hace que las aguas suban y provoquen inundaciones en ciertos casos y que además, favorece la entrada de especies o bacterias foráneas a los ecosistemas, lo que fuerza las migraciones e incluso la extinción de especies.

Otra amenaza es la acidificación marina, que en términos prácticos no es más que la contaminación de las aguas del océano con $\mathrm{CO}_{2}$. Finalmente, está la erosión costera que aqueja a las playas, debido a la desintegración de las rocas provocadas por las olas como efecto de las mareas y corrientes marinas.

Cartagena, Barranquilla, Santa Marta y Buenaventura tienen el mayor riesgo por cuenta de la erosión de sus playas y las construcciones cercanas al mar. En esto coincide Diana Moreno, encargada de la Dirección de Asuntos Marinos Costeros y Recursos Acuáticos (Damcra) del Ministerio de Ambiente y Desarrollo Sostenible. "El 42 por ciento de las costas en la región Caribe y otro 17 por ciento en la región Pacífico, presentan problemas de erosión costera, situación que se ha venido incrementando en los últimos años, en parte debido a la 
Ensino, Saúde e Ambiente - Número Especial, pp. 389-411, Junho. 2020

improvisación e implementación de soluciones individualizadas que no cuentan con los estudios técnicos necesarios, ni un análisis integral”.

Sobre este tema, Constanza Ricaurte, coordinadora del programa de Geociencias Marinas y Costeras del Instituto de Investigaciones Marinas y Costeras (Invemar), indica que los análisis incluidos en la Tercera Comunicación Nacional de Cambio Climático arrojan cifras nada alentadoras: en 2040 la región Caribe perdería 5.049 hectáreas de playa y 12.827 en 2070; mientras que en la región Pacífico se perderían 7.579 en 2040 y 26.117 en 2070.

\section{Movimiento Popular}

\subsection{El movimiento popular y el contexto climático.}

Dentro del contexto de eventos extremos, como se vio anteriormente, a partir de los datos de informes de PBMC, existe una alta posibilidad de cataclismos como olas de calor, fuertes lluvias y consecuentemente deslizamientos de tierra. De esto, una injusticia climática es notable. Según lo previsto por varios informes de la Convención Marco de las Naciones Unidas sobre el Cambio Climático (CMNUCC), el Panel Intergubernamental sobre el Cambio Climático (IPCC) y entre otros, uno de los argumentos para Contener las emisiones de GEI es sin duda la vulnerabilidad de los países en desarrollo. Existe una contradicción entre los países del norte, que tienen el mayor acceso al consumo de energía, materias primas y otros, que los países en desarrollo. Según el conocimiento científico, debido al cambio climático, se afirma mucho que las poblaciones ricas consumen más recursos y energía, y sufren menos los efectos del cambio climático.

Los defensores de la justicia climática argumentan que aquellos que son menos responsables de las emisiones de gases de efecto invernadero serán los que más sufrirán los impactos del cambio climático. Para tratar de minimizar estos problemas, proponen que se implementen iniciativas y políticas que aborden las dimensiones éticas de los derechos humanos del cambio climático para reducir la vulnerabilidad de los grupos sociales desproporcionadamente afectados por el cambio climático (EBI, 2009; ROBERTS; PARKS, 2009; SHEPARD; CORBIN-MARK, 2009; TYREE; GREENLEAF, 2009 apud MILANEZ, B .; FONSECA, I.F., 2011).

Sin embargo, el sujeto que disfruta poco o nada paga un alto precio de supervivencia en vista de eventos extremos. Al igual que la población pobre representada por una gran 
Ensino, Saúde e Ambiente - Número Especial, pp. 389-411, Junho. 2020

proporción de negros, marrones e indígenas, que disfrutan de las cualidades más bajas de la vida.

Para comprender la crisis ecológica global, es importante comprender que la intoxicación que sufren los afroamericanos en el centro-sur de Los Ángeles y los mexicanos en los fabricantes de automóviles fronterizos de EE. UU. Tiene sus raíces en el mismo sistema de explotación económica, opresión racial y devaluación de la vida y lo humano La búsqueda de soluciones a los problemas ambientales y las formas de lograr el desarrollo sostenible en los Estados Unidos tiene implicaciones considerables para el movimiento ambiental mundial (Bullard, 1996. p.2).

En los grandes centros urbanos, la ciudad de Río de Janeiro, más específicamente, las áreas mejor estructuradas, que se puede decir que están pavimentadas, reflejan poco impacto en la población debido a un cataclismo ambiental. Sin embargo, la población negra circundante, agregada por barrios marginales, sufre más allá de las inundaciones. Como se puede ver en el mapa de Young y Santos (2010), hay datos que proyectan una consecuencia futura de fuertes lluvias en los municipios del estado. Las regiones marcadas en rojo corresponden a los barrios marginales de varios municipios; La cromatografía grisácea atribuida a las clases alta, media y baja corresponde a los índices de intensidad de las tres bandas.

En naranja claro, áreas con dimensiones de hasta 0,40 m; en naranja oscuro, aquellos con dimensiones entre 0,40 my 0,60 m; y, en marrón, las áreas con dimensiones de 0,60 ma 1,50 m. Este último representa las posibles áreas alcanzadas por el aumento del nivel del mar, teniendo en cuenta las oscilaciones del mar, que pueden alcanzar los 0,80 m. Cabe señalar que la cantidad de áreas afectadas es acumulativa, es decir, la cuota de hasta $1.50 \mathrm{~m}$ incluye la cuota de hasta $0.40 \mathrm{~m}$ y $0.60 \mathrm{~m}$, así como la cuota de hasta $0.60 \mathrm{~m}$ incluye la cuota de hasta 0.40m (IPP, 2008 apud MARENGO, JA; SCARANO, FR, 2016). 
Ensino, Saúde e Ambiente - Número Especial, pp. 389-411, Junho. 2020

Figura 1 - Mapa adaptado de vulnerabilidad al cambio climático en los municipios del estado de Río de Janeiro.

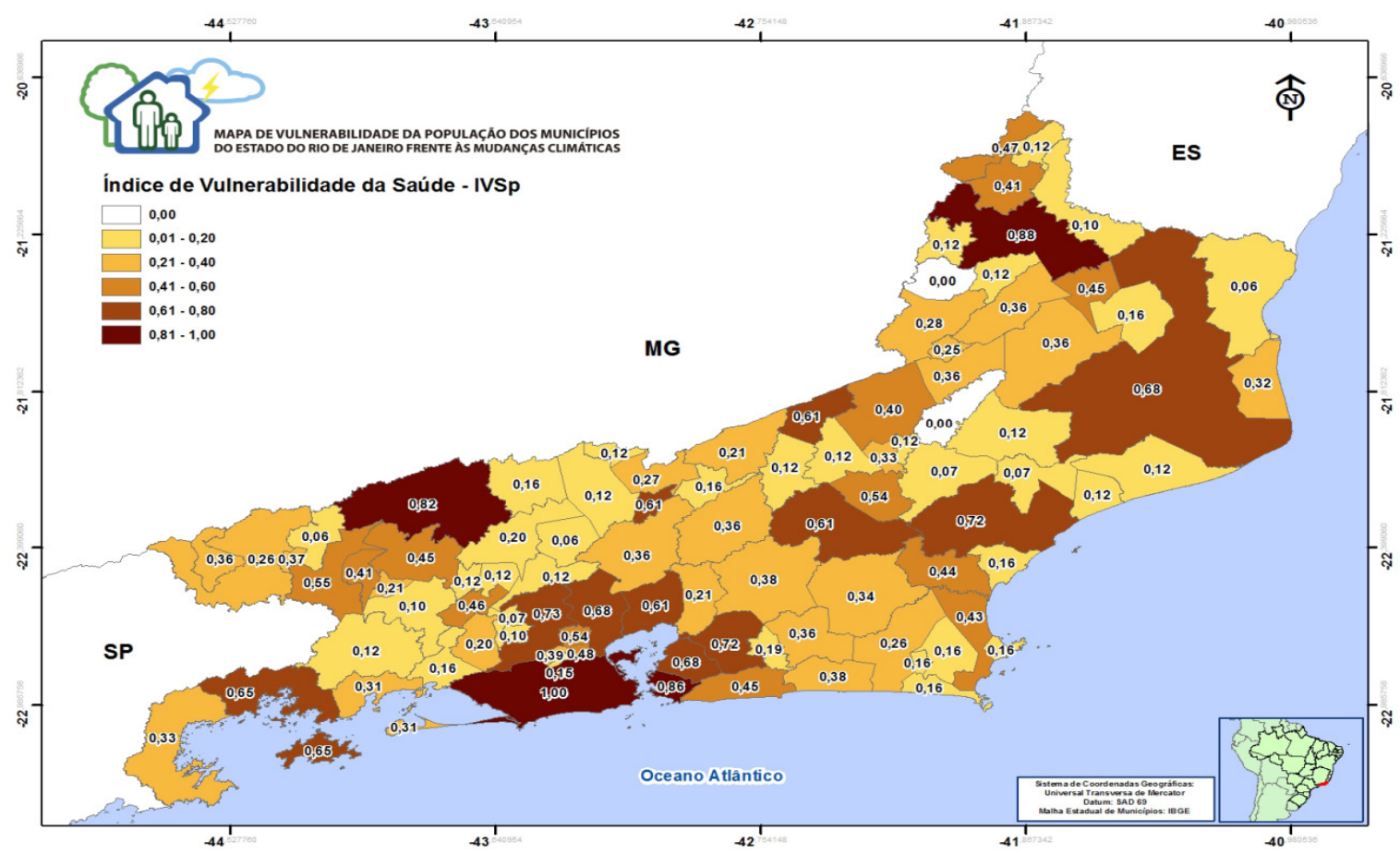

Fuente: FIOCRUZ, 2014.

La desigualdad social, junto con la concentración de ingresos en los grandes centros urbanos, están atrayendo a la población menos favorecida en ese mismo lugar en busca de un mayor poder económico. En esto, se generan ocupaciones irregulares como los barrios marginales. Procedente de la supresión generalizada de plantas que conduce a la construcción irregular, esta población es víctima de la vulnerabilidad social y ambiental, en vista de las consecuencias de los eventos climáticos más allá de la pobreza. "Con el advenimiento del neoliberalismo, en la década de 1990, la ola de privatización de las empresas estatales y los recortes en el gasto público en salud y educación, que representa el fin de un estado de bienestar que ni siquiera se consolidó en Brasil.

Hubo un aumento significativo del desempleo y la consiguiente precariedad del trabajo (DAMASCENO, E. S.; SANTANA JUNIOR, H. A., 2011). Junto con el cambio climático, las ocupaciones irregulares y el asfalto contribuyen a los efectos del calor extremo y también son víctimas de lluvias extremas que provocan erosión y matan a muchos de los residentes de los alrededores. Al reconocer el tema de los barrios bajos, se identifica a sí mismo como personas en su mayoría negras, con baja educación y extrema pobreza, siendo enmarcado como un sujeto sujeto de desigualdades. 
Ensino, Saúde e Ambiente - Número Especial, pp. 389-411, Junho. 2020

Para ilustrar el informe anterior, la ciudad de Río de Janeiro pasó por varios períodos de fuertes lluvias en 2010 y 2011. En el período del 5 y 6 de abril de 2010, el Estado fue víctima de este evento extremo, en el barrio marginal mejor conocido como Morro dos Prazeres, en el barrio de Santa Teresa del centro de Río de Janeiro, fue víctima de la llegada del cambio climático. La fuerte lluvia causó sucesivos deslizamientos de tierra que mataron a 30 víctimas. En el mismo período, la comunidad de Guararapes, en Cosme Velho, sufrió deslizamientos de tierra, dejando 4 muertos; ambos clasificados por el estudio, "Los 50 Accidentes Geológico-Geoténicos más grandes de la Ciudad de Río de Janeiro entre 1966 y 2016 ” del Ayuntamiento de Río de Janeiro, como eventos de Clase III, que se refiere a un nivel altamente expresivo según una escala de puntuación. Afectados por la misma lluvia, en la noche del 7 de abril de 2010, Morro do Bumba mató a 267 personas el 7 de abril de 2010, hoy la antigua comunidad se conoce como "Cementerio".

El número de tales casos podría ser aún mayor, ya que familias enteras fueron enterradas, sin sobrevivientes para reclamar los cuerpos. Un residente de la comunidad por más de 30 años, Joel Gomes, de 53 años, conocía a los 45 muertos rescatados de la inundación que barrió la Bumba. Su casa es una de las pocas que resistió en la ladera, que ahora está cubierta de césped verde. (Disponible en: https://www.terra.com.br/noticias/brasil/cidades/apos-1-ano-da-tragedy-morro-do-bumba-and-calledcemiterio, ed4aaf17b94fa310VgnCLD200000bbcceb0aRCRD.html, )

En 2011, la comunidad Ant también fue afectada. Incluso hoy en día, sus residentes, además de estar estructuralmente afectados, se ven sacudidos psicológicamente debido a la pérdida material y física, en el caso de familiares, amigos y vecinos que viven en la misma comunidad. Hasta el día de hoy, estas personas no han cumplido con la ley y las políticas públicas garantizadas para su dignidad de vida. Posteriormente, lo que apoyó un movimiento de resiliencia en Morro da Formiga fue el programa de "Reforestación Mutirão" que existe desde 1997. En él, los residentes son utilizados como fuerza laboral para la acción ambiental, en la que se planta vegetación forestal. Atlantic como una forma de satisfacer la demanda de bajas emisiones de carbono y las medidas compensatorias elaboradas por los Acuerdos Internacionales de la ONU.

Las acciones actuales desarrolladas por el Ayuntamiento en Morro da Formiga son parte del Programa Social Río + coordinado por el Instituto Pereira Passos en colaboración con ONU-Hábitat. ONU-Hábitat apoya al Instituto Pereira Passos en la gestión general del 
Ensino, Saúde e Ambiente - Número Especial, pp. 389-411, Junho. 2020

programa y en la producción de datos, análisis y seguimiento de cada territorio de Río + Social (KASSIADOU, A. et al., 2018).

Este año, el 10 de noviembre de 2018, ocurrió la tragedia ambiental en la favela de Boa Esperança en Niterói. Aunque el Sistema de Defensa Civil de Niterói ha sido reconocido como uno de los cinco mejores en Brasil, el área es vista por muchos expertos como un área insegura y debe insertarse en la actualización de las áreas de riesgo. La tragedia contó con 14 muertos y 11 rescatados.

Más de 200 profesionales están presentes en el trabajo de ayuda y la búsqueda bajo los escombros, así como muchos voluntarios. También hay un análisis del riesgo de nuevos deslizamientos de tierra y la Defensa Civil informó que la región aún es peligrosa y necesita monitoreo (Disponible en: https://www.anf.org.br/tragedia-na-favela-boa-esperanca/; https://www.anf.org.br/o-desastre-socioambiental-em-boa-esperanca /).

\subsection{Conflicto armado y desplazamiento como impulsores de la sensibilidad al cambio climático en Colombia}

Colombia registra una de las tasas más altas en el mundo de personas en situación de desplazamiento interno (PSDI) como resultado de décadas de conflicto civil, aumentando la sensibilidad de la población a los impactos del cambio climático. En Colombia, el desplazamiento forzado interno sigue siendo motivo de preocupación, pese a los esfuerzos del gobierno para negociar la solución pacífica del conflicto con las Fuerzas Armadas Revolucionarias de Colombia (FARC) y para mejorar la respuesta oficial más amplia al problema. Según la Agencia de las Naciones Unidas para los Refugiados (ACNUR), la inseguridad y la violencia continúan azotando a muchas regiones, tanto en las zonas urbanas como en las rurales. Según cifras oficiales, desde 2000 se registraron más de 4.7 millones de personas en situación de desplazamiento interno, aunque es posible que la cifra real sea muchísimo más elevada. El cambio climático puede tener consecuencias graves para las PSDI. Una vez desplazadas, las personas tienen que soportar la perdida de sus medios de subsistencia y la pobreza y exclusión económica. En Colombia, la gran mayoría de PSDI se desplaza a zonas urbanas, donde se ven obligadas a habitar en vecindarios marginales muy expuestos a los riesgos de desastres naturales, como sismos, inundaciones y deslizamientos de tierra. Por ejemplo, Bogotá acoge a muchas PSDI, y es una ciudad propensa a los temblores, inundaciones e incendios forestales. También es probable que las comunidades urbanas 
pobres que albergan a los desplazados se encuentren en condiciones de hacinamiento y carezcan de servicios adecuados de acueducto, alcantarillado y de saneamiento acrecentando, por tanto, los riesgos de seguridad y protección. Por ejemplo, en Colombia, las altas tasas de deforestación en los asentamientos informales y sus alrededores pueden acrecentar la inestabilidad de las laderas, la erosión del suelo y la frecuencia de inundaciones repentinas y deslizamientos de tierra provocados por la precipitación (CAF, 2014).

\section{Consideraciones finales}

El concepto de antropoceno como etapa geológica de la tierra en la que el ser humano está alterando la geomorfología, los ciclos biogeoquímicos, entre otros, en la actualidad ha alcanzado también a las ciencias naturales y a maneras disciplinarias de producir conocimiento, al igual que a los debates públicos y las políticas públicas relacionadas con lo global-local. Su trascendencia se debe en parte a que el concepto emerge en las ciencias geológicas (ciencias de la tierra) y tiene aceptación en diversos ámbitos académicos, aun entre los teóricos críticos de las ciencias humanas.

El capitaloceno por su parte, es una corriente de pensamiento surgida desde la ecología política principalmente, en la cual se afirma que no todos los seres humanos tenemos igual responsabilidad en el cambio climático de nuestro planeta, son los países y los grandes empresarios y multinacionales quienes por la necesidad de obtener mayor cantidad de materia y energía, para los procesos de producción y acumulación de capital, están destruyendo o alterando al menos el equilibrio dinámico de la tierra.

Con las reflexiones sobre el capitaloceno como principal factor destructor del equilibrio dinámico de nuestro planeta, queda manifiesto el indudable y evidente racismo e injusticia ambiental al que los grandes capitales y gobernantes someten y exponen a minorías, generalmente de escasos ingresos o étnicas. Los pasivos ambientales no son asumidos de manera equitativa o preferiblemente proporcional al grado de contaminación o alteración de los ecosistemas que se realice, por el contrario, los países del sur o del Abya Ayala, seguimos siendo el patio trasero y el sumidero de las grandes potencias, a la vez que representamos un valor estratégico por la inmensa abundancia de patrimonio natural, cultura y social.

Teniendo en cuenta que las personas no blancas resultan en una suma considerablemente mayor que la población blanca, lo que configura estas zonas de sacrificio de las localidades. A través de los datos seleccionados, el estado en general tiende a sufrir los efectos adversos del cambio climático. Sin embargo, se observa que las regiones marcadas ISSN 1983-7011 
tienen una demanda de políticas públicas que dialoguen con la cosmología de la población afectada. Ante esto, es necesaria una EA crítica y descolonial en la que pueda asociarse con ella.

Teniendo en cuenta lo que se ha visto, una alta proporción del territorio de Río de Janeiro, que ya tiene una vulnerabilidad general en términos de salud, condiciones económicas y ambientales per se, son los lugares más habitados por la población no blanca. A partir de esto, se configura en el mismo, el "racismo ambiental" que se denunciará a través de cataclismos ambientales, como los efectos de inundaciones, movimientos de masas, deslizamientos de tierra y extremos de calor.

\section{REFERENCIAS BIBLIOGRAFICAS}

ACSELRAD, H. Justiça Ambiental: novas articulações entre meio ambiente e democracia. Movimento Sindical e Defesa do Meio Ambiente: o debate internacional, série Sindicalismo e Justiça Ambiental, IBASE; CUTRJ; IPPUR-UFRJ, Rio de Janeiro, v.3, p. 7-12, 2000.

AGYEMAN, J. y B. Evans. 'Just sustainability': the emerging discourse of environmental justice in Britain. The Geographical Journal 170:155-164. 2004.

ARRIAGA L., Alicia. Justicia Ambiental. El estado de la cuestión. Revista Internacional de Sociología (RIS). Vol.69, $\mathrm{n}^{\circ}$ 3, Septiembre-Diciembre, 627-648, 2011. ISSN: 0034-9712. DOI:10.3989/ris.2009.12.210. 2009.

BARROZO, L. A.; SANCHÉZ, C. Educação Ambiental Crítica, Interculturalidade e Justiça Ambiental: entrelaçando possibilidades. In: Encontro Pesquisa em Educação Ambiental, 8, 2015, Rio de Janeiro. Disponível em: <http://epea.tmp.br/epea2015_anais/pdfs/plenary/139.pdf>. Acesso em: 08 dez. 2018.

BORRÁS S. Movimientos para la justicia climática global: replanteando el escenario internacional del cambio climático. Relaciones Internacionales Número 33 • Octubre 2016 Grupo de Estudios de Relaciones Internacionales (GERI) - UAM. Enero 2017.

BRASIL. Ministério da Ciência, Tecnologia e Inovação. Secretaria de Políticas e Programas de Pesquisa e Desenvolvimento. Coordenação-Geral de Mudanças Globais de Clima. Modelagem climática e vulnerabilidades Setoriais à mudança do clima no Brasil. Brasília, DF, n. 2, p.590, 2016.

BRASIL. Comitê Interministerial sobre Mudança do Clima. Decreto $\mathrm{n}^{\circ}$ 6.263, de 21 de novembro de 2007. Plano Nacional de Mudança do Clima. Brasília, DF, 2009.

BULLARD, R. D. Anatomia do racismo ambiental e o movimento por justiça ambiental. In: BULLARD, R. D. (Org.). Confronting Environmental Racism: voices from the Grassroots. Boston: South End Press. 1996.

CANO, R, O. E. Capitaloceno y adaptación elitista. Revista Ecología Política. p. 4. 2017.

CATARINO, C. S. A centralidade do Racismo Ambiental na abordagem crítica da Educação 
Ambiental. Trabalho de Conclusão de Curso (Graduação em Ciências Biológicas) Universidade Federal do Estado do Rio de Janeiro. 2016. Disponível em: $<$ http://www.fase.org.br/projetos/clientes/noar/noar/UserFiles/17/File/AnatomiadoRaci smoAmb.pdf.>. Acesso em: $01 \mathrm{dez}$.

COLECTIVO EL KINTRAL KILTRX SUBALTERNX. Los Cuadernos del Capitaloceno. Norte Semiárido - Primavera 2018- $\mathrm{N}^{\mathrm{o}}$ 1. Pag 13. 2018. Disponible en http://www.mapuexpress.org/wp-content/uploads/2018/11/CapitalocenoI.pdf..

GUTIÉRREZ, A., A. En el camino de la justicia ambiental: estableciendo vínculos entre medio ambiente y justicia social Revista de Ciencias Sociales (cr), vol. Iv, núm. 146, 2014, pp. 113-125. Universidad de Costa Rica, San José, Costa Rica. 2014.

HELFAND, G. E. y L. J. PEYTON. 1999. "A Conceptual Model of Environmental Justice.” Social Science Quarterly 80: 68-83.

KASSIADOU, A. et. al. (Orgs.). 2018. Educação Ambiental Desde EI Sur. Macaé: Editora Nupem, 2018.

LEVETT, P. N. Leptospirosis. Clinical Microbiology Reviews, Washington, DC, v.14, n. 2, p. 296-325, 2001. In: BRASIL, Ministério da Ciência, Tecnologia e Inovação. Modelagem climática e vulnerabilidades Setoriais à mudança do clima no Brasil. Brasília, DF: Ministério da Ciência, Tecnologia e Inovação, 2016.

MAGRIN, G. O.; MARENGO, J. A.; BOULANGER J. P. Central and South America. 2014. In: Climate Change 2014: Impacts, Adaptation, and Vulnerability. Part B: Regional Aspects. [BARROS, V. R.; FIELD, D. J.; DOKKEN, M. D. et. al. [Eds.]. In: MARENGO J. A.; SCARANO F. R. [Eds.]. Impacto, vulnerabilidade e adaptação das cidades costeiras brasileiras às mudanças climáticas. Relatório Especial do Painel Brasileiro de Mudanças Climáticas. Rio de Janeiro: PBMC; COPPE - UFRJ, 2016.

MALAGÓN-ROJAS, J. N., GARROTE-WILCHES, C. F., \& Castilla-Bello, P. A. Cambio climático y salud humana: una revisión desde la perspectiva colombiana. Salud Uninorte, 33(2), 224-241. 2017.

MILANEZ, B.; FONSECA, I.F. Justiça Climática e Eventos Climáticos Extremos: O Caso das Enchentes no Brasil. Centro de Estudos de Desigualdade e Desenvolvimento, Universidade Federal Fluminense, Niterói, Rio de Janeiro, n. 38, 2011. Disponível em: <www.proac.uff.br/cede>. Acesso em: 07 dez. 2018.

SEMANA SOSTENIBLE, 20117. Las playas de Colombia, en riesgo por el cambio climático. impacto 2017/11/02. rescatado de https://sostenibilidad.semana.com/impacto/articulo/cambioclimatico-amenaza-las-playas-de-colombia/38901

MARINHO, D. P. et. al. Índice de vulnerabilidade às mudanças do clima nos municípios do estado do Rio de Janeiro. 2014. In: BRASIL, Ministério da Ciência, Tecnologia e Inovação. Modelagem climática e vulnerabilidades Setoriais à mudança do clima no Brasil. Brasília, DF: Ministério da Ciência, Tecnologia e Inovação, 2016.

PÉREZ, R., M. A. Conflictos ambientales en Colombia: Inventario, caracterización y Análisis Estudio para 72 casos de injusticia ambiental. Universidad del Valle - Instituto CINARA, Cali, Colombia. Pag 70. 2014.

PINEDA C, MUNOZ-LOUIS R, CABALLERO-URIBE CV, VIASUS D. Chikungunya in the region of the Americas. A challenge for rheumatologists and health care systems. Clinical rheumatology;35(10):2381-5. 2016. 
RAMÍREZ G. S.J.; GALINDO, M, M, G.; CONTRERAS S. C. Justicia ambiental. Entre la utopía y la realidad social. Universidad Autónoma de San Luis Potosí. Época II - Vol. III Núm. 1. ISSN 1870-1191. 2015.

RUEDA K, TRUJILlO JE, CARRANZA JC, VALLEJO GA. Transmisión oral de Trypanosoma cruzi : una nueva situación epidemiológica de la enfermedad de Chagas en Colombia y otros países suramericanos. Biomédica; 34:631-41. 2014.

SILVA, J. V. C. Sociedades de água do morro da Formiga: subsídios para Educação Ambiental de base comunitária e ecologia de saberes em uma favela carioca. Dissertação (Mestrado em Educação) - Programa de Pós-Graduação em Educação, Universidade Federal do Estado do Rio de Janeiro, Rio de Janeiro. 2016.

TOLEDO M. V. 2019. ¿? Qué es el capitaloceno Recuperado de la revista: Desde abajo, https://www.desdeabajo.info/ambiente/item/36539-que-es-el-capitaloceno.html.

ULLOA, A. Dinámicas ambientales y extractivas en el siglo XXI: ¿es la época del Antropoceno o del Capitaloceno en Latinoamérica? Desacatos 54, mayo-agosto 2017, pp. 5873. 2017. 